\title{
Les défis partagés du gouvernement du Québec et de son industrie forestière ${ }^{1}$
}

\author{
par M. Jacques Robitaille ${ }^{2}$
}

\section{Introduction}

Au risque de rappeler de vieux clichés connus, il m'apparait essentiel, en guise d'introduction, de rappeler les principaux éléments qui caractérisent la ressource forestière québécoise et son secteur industriel forestier.

La forêt productive québécoise couvre une superficie de $516000 \mathrm{~km}^{2}$, représentant ainsi $20 \%$ des forêts canadiennes et $2 \%$ des forêts du globe. Le territoire se partage à raison de $90 \%$ en forêt publique et $10 \%$ en forêt privée, cette dernière fournissant par contre $24 \%$ des approvisionnements de l'industrie.

La possibilité annuelle à rendement soutenu s'établi à 57,3 millions de mètres cubes. Les résineux représentent $65 \%$ de ce volume alors que les feuillus y contribuent pour $35 \%$.

Peu publicisée et souvent de fois oubliée, l'industrie forestière québécoise constitue un des éléments clés de notre économie, tant au plan des emplois, de la valeur des expéditions que des investissements. Mais plus encore, son étalement sur le territoire québécois en fait une composante essentielle pour assurer le développement, sur une base durable, de nos régions. Essayons d'imaginer, sans l'apport du secteur forestier, des villes comme Chandler, La Tuque, Amos, etc. - je pourrais vous en énumérer ainsi plus de 250 .

Le Québec est ainsi la deuxième province, après la ColombieBritannique, pour l'importance de son industrie forestière. Le Québec occupe cependant le premier rang pour la valeur des expéditions de pâtes et papiers avec $33 \%$ du total canadien et $44 \%$ dans le cas du papier journal.

Pour ce qui est des produits du bois, le Québec vient second pour le sciage résineux avec $22 \%$ de la production canadienne en 1995, en plus d'avoir une importante représentation dans l'industrie du panneau gaufré, du particule et du MDF.

En termes concrets, c'est plus de 16,9 milliards de dollars d'expéditions, 62000 emplois directs et une contribution nette de 8,8 milliards de dollars à la balance commerciale. Le secteur des pâtes et papiers représente $77 \%$ des ventes de l'industrie des produits forestiers.

\section{Un secteur en mutation}

Tous s'accordent pour reconnaitre que nous vivons dans un monde en profonde mutation et le secteur forestier n'échappe pas à cette réalité. La multiplicité et l'accélération des changements qui nous assaillent nous obligent à revoir notre perception des choses, nos façons de faire et même nos raisons d'être. Plus jamais, les choses ne seront comme avant.

Je m'attarderai ici à explorer avec vous trois aspects des changements qui devront, dans le futur, conditionner l'action et les décisions, à la fois de l'industrie et du gouvernement. Il s'agit de la ressource et de l'évolution de sa consommation, l'environnement commercial et finalement, le régime forestier québécois.

\footnotetext{
${ }^{1}$ Presentation to the Price Waterhouse Forest Industry Conference, 26 November 1996, Montréal, Québec.

${ }^{2}$ Sous-Ministre Associé aux forêts, Ministère des Ressources naturelles, 880 Chemin Ste-Foy, Québec (Québec) G1S 4X4.
}

\subsection{La ressource et sa consommation}

Globalement, le secteur industriel forestier québécois a connu une importante croissance au cours des cinq dernières années, avec le résultat net que la consommation de fibre s'est accrue de façon substantielle.

Avec une consommation totale de 40,5 millions de mètres cubes de bois rond, la presque totalité des disponibilités de résineux économiquement exploitables à rendement soutenu des forêts québécoises sont présentement utilisées. Les résineux représentent $80,5 \%$ de ce volume et la forêt publique fournit $68,4 \%$ de ces bois, le reste provenant des forêts privées et des bois d'importation. Il faut mentionner que ces volumes génèrent 20 millions de mètres cubes de sous-produits comme les copeaux, sciures et planures qui alimentent l'industrie des pâtes et papiers ainsi que celle du panneau aggloméré.

De façon plus précise, les disponibilités en fibre pouvant appuyer la mise en place de nouvelles capacités de production ne se retrouvent plus, à toutes fins pratiques, que dans la fibre de qualité pâte dans les essences de feuillus durs. Pour quelques décennies à venir, l'expansion de l'industrie forestière au Québec devra passer par la seconde transformation et une utilisation plus complète de la fibre (meilleure récupération).

Cette observation nous amène à faire d'autres constats. Le taux de récupération de la fibre s'est accru de façon importante avec la conséquence qu'on transforme maintenant, la technologie aidant, des bois de beaucoup plus petites dimensions et ces bois sont récoltés à des distances de plus en plus grandes. Ce faisant, le facteur coût devient plus critique. Il s'agit d'un élément qui a d'ailleurs été abondamment défendu auprès des Américains dans le cadre du dossier du bois d'oeuvre.

Un autre élément de changement important s'est opéré au cours des dernières années en terme de consommation de la fibre. Il s'agit de la diminution radicale de consommation de bois rond par l'industrie des pâtes et papiers. La consommation de billes résineuses a en effet décru de $4,2 \mathrm{Mm}^{3}(54,5 \%)$ alors que celle de billes de feuillus s'est accrue de $350000 \mathrm{~m}^{3}(13,7 \%)$ entre 1991 et 1995. Évidemment, ce changement s'est traduit par une augmentation équivalente de la consommation de copeaux $(35,7 \%)$.

$\mathrm{Ce}$ changement majeur origine de deux facteurs principaux. D'abord, le remplacement de la technologie de défibrage aux meules par celui du raffinage thermomécanique et ce, afin de réduire les coûts d'approvisionnement en fibre et augmenter la qualité de la pâte.

Le second facteur découle de la décision du gouvernement américain d'imposer un contenu minimal en fibre recyclée pour le papier journal. L'industrie des pâtes et papiers a donc investi depuis 1992, $340 \mathrm{M} \$$ pour la construction de six ateliers de désencrage pour une capacité totale annuelle de 615000 tonnes.

Pour cette période, bien que le niveau de production de l'industrie des pâtes et papiers soit resté relativement stable, ceux du sciage et du panneau aggloméré ont connu une croissance sans précédent. La consommation de l'industrie du sciage s'est donc 
accrue de $50 \%$, dont $77,4 \%$ dans le cas du sciage feuillu. Ainsi, c'est $6 \mathrm{Mm}^{3}$ de plus qui a été attribué à l'industrie du sciage résineux. Quant à l'industrie du panneau aggloméré, c'est le OSB qui accapare la part du lion avec une croissance de $64 \%$ de sa consommation de bois rond.

Cette évolution amène d'autres constats qui sont à la base même d'importants changements structuraux, en plus de conditionner le développement futur de l'industrie forestière québécoise.

Nous évoluons donc maintenant dans un contexte local de rareté relative de fibre pour les besoins futurs et d'équilibre très serré pour les besoins présents. Cet équilibre rend interdépendantes l'une de l'autre l'industrie des pâtes et papiers et celle du sciage. Nous pouvons ainsi passer en très peu de temps, selon l'évolution des cycles de chacune de ces industries, d'une quasi pénurie de copeaux à d'importants surplus et cela, en quelques mois. L'industrie des pâtes et papiers a réagi à cette situation en poursuivant l'intégration de l'industrie du sciage à sa structure. Cette intégration qui atteint maintenant $70 \%$, n'est pas sans conséquence sur la fluidité du marché des copeaux.

Globalement, cette situation fragilise l'équilibre du marché de la fibre au Québec rendant ainsi les petites entreprises non intégrées plus vulnérables et moins en mesure de compétitionner pour l'obtention de leurs fibres ou pour disposer de leurs sous-produits, en particulier les copeaux.

Pour avoir une image complète, il faut aussi mettre en perspective l'état de notre disponibilité en fibre avec la situation qui prévaut au plan international. Il faut en effet constater qu'à l'échelle du globe, nous sommes actuellement en surplus de fibre. Les développements futurs de nos compétiteurs se feront là où cette fibre sera disponible et, sans doute, dans certains des crénaux qui sont la base de nos productions et ce, à des coûts hors de notre portée. Voilà une situation préoccupante.

\subsection{L'environnement commercial}

Encore ici, le moins que l'on puisse dire, c'est que les choses ont bien changé. Pendant longtemps, forts de la qualité et de l'abondance de notre fibre, nous avons été les rois du papier journal et, plus récemment, du bois d'oeuvre. Ce n'est plus le cas. D'autres arrivent à faire ces produits et nous sortent du marché par une compétitivité supérieure, dans le cas du papier journal, et par l'imposition de quotas, dans le cas du bois d'oeuvre.

La vulnérabilité de notre secteur des pâtes et papiers est apparue de façon particulièrement évidente lors du dernier creux cyclique du début des années 1990. Bien que le secteur ait réussi, à la faveur du dernier cycle haussier, à améliorer sa position concurrentielle, plusieurs unités de production demeurent encore à l'autre extrémité de l'échelle de productivité.

Le marché des commodités est maintenant à la portée de compétiteurs pour lesquels nos avantages comparatifs traditionnels ne valent plus. Les problèmes sont multiples; la nature de nos produits (commodités), notre parc technologique et notre dépendance de technologies étrangères pour les pâtes et papiers et le panneau, notre manque d'agressivité à développer des nouveaux marchés, notre indifférence vis-à-vis la $R / D$, nos efforts en ce domaine atteignent à peine la moitié de ce que font les Scandinaves et le tiers de celui de nos voisins du sud (USA).

Certains diront, en pointant les redevances forestières, «il y a aussi le coût de la fibre». Cela m'apparaît trop facile comme prétexte. Malgré les éléments que j'ai mentionnés précédemment, le coût de la fibre au Québec demeure encore compéti- tif si on le compare aux autres provinces canadiennes, aux états américains (sauf pour les états du Sud), aux pays scandinaves ou aux pays européens. Tous ces états ont une chose en commun: ils appliquent, à des degrés divers, les standards actuellement les plus évolués en matière de gestion forestière. Les pays qui, actuellement nous battent sur le coût de la fibre, n'en sont pas encore rendus là. Par contre, il ne fait aucun doute que les exigences actuelles et futures, en matière de gestion forestière, les dirigent dans cette direction. À nous de jouer nos cartes en conséquence et de transformer à notre avantage cette situation. J'y reviendrai plus tard.

\subsection{Le régime forestier québécois}

Cela m'amène maintenant à vous parler du régime forestier québécois et de son évolution. En effet, depuis 1986, le gouvernement du Québec a mis en place une série de mesures réglementaires et administratives régissant la gestion forestière, afin de répondre aux attentes de la population du Québec, propriétaire de cette richesse collective qu'est la forêt publique, ainsi qu'aux exigences des conventions internationales en matière de développement durable.

Ce régime s'appuie sur une série de principes, tels:

- le respect de la capacité de la forêt à produire;

- la protection des composantes du milieu forestier et de ses ressources;

- l'utilisation harmonieuse de l'ensemble des ressources du milieu forestier;

- une gestion transparente.

Nous avons orienté l'évolution du régime forestier pour tenir compte des nouveaux enjeux tels la protection de la biodiversité, les critères reconnus de développement durable et une réponse aux demandes des populations locales de participer à la gestion de cette ressource. Nous sommes ainsi en mesure de réfuter sur les tribunes internationales les attaques de nos dénigreurs.

\section{Les défis que nous devrons relever}

\subsection{Gestion de la ressource}

$\mathrm{Au}$-delà d'un mot à la mode, la gestion durable des forêts est devenue un impératif de marché. D'abord placé au feuilleton des rencontres internationales en matière d'environnement (Sommet de Rio, etc.), ce concept a rapidement été repris par les groupes de pression environnementaux pour ensuite être utilisé comme un élément discriminant sur certains marchés.

Le terme générique du concept a rapidement pris une connotation particulière en matière de gestion forestière. La perception très écologique du milieu forestier qu'ont en général les citoyens a rapidement été mise en opposition avec les images dévastatrices de nos pratiques prévalant jusqu'alors. Des campagnes habilement orchestrées, conjugées à notre attitude passive d'alors, ont très rapidement permis à ce phénomène de dégénérer en arme commerciale.

Mais au-delà de cette dimension, l'évolution des préoccupations environnementales commande maintenant une nouvelle éthique en matière de gestion forestière. Nous avons su nous adapter à ces nouvelles règles et nous avons au Québec un régime forestier qui permettra sans doute à notre industrie de passer le test de la certification environnementale. 
Il nous faut dependant demeurer attentif à ce dossier et s'assurer que nous pourrons maintenir la qualité de notre gestion forestière. Au-delà des exigences reliées à nos marchés, il faut se rappeler que la majorité des forêts du Québec sont de propriété publique. Nous ne pouvons faire fi des aspirations de la population à un usage polyvalent et de qualité, de ce qui, en plus d'être un pourvoyeur d'emploi, est aussi un milieu de vie.

Les aspirations de la population à cet effet sont très variables d'une communauté à l'autre. On n'a qu'à penser aux contraintes reliées aux demandes des populations autochtones. Il s'agit d'une réalité à la fois légitime et incontournable. L'ignorer fait courir, à cette industrie, un grand danger, on n'a qu'à se rappeler le dossier Clayoquot Sound en Colombie-Britannique et le récent référendum du Maine sur les coupes à blanc.

Nos interventions futures en forêt devront donc être exemplaires. De plus, notre gestion devra être transparente, c'est une condition essentielle pour établir notre crédibilité et la relation de confiance qui devrait en découler. Nous devrons, de plus, continuer à développer nos habiletés et outils afin de bien informer la population et nos clients sur la qualité de notre gestion forestière. Il s'agit là d'un domaine où nous avons tardé à nous impliquer.

Au-delà de la dimension environnementale, il y a aussi toute la question de la productivité des forêts et de l'aménagement de celles-ci. À venir jusqu'à présent, nous avons bénéficié de la générosité de la nature n'ayant qu'à récolter ce que celle-ci avait mis à notre disposition. Déjà , notre implication s'est accrue depuis l'avènement du régime forestier actuel permettant ainsi de faire passer notre rendement soutenu en SEPM de $18 \mathrm{Mm}^{3}$ à $30 \mathrm{Mm}^{3}$. Nous sommes encore bien loin de l'utilisation du plein potentiel de production de nos forêts que pourrait permettre une sylviculture plus intensive.

La présente situation de disponibilité de matière ligneuse limite les développements futurs de l'industrie en terme d'augmentation de capacité. De plus, les effets potentiels des incendies forestiers et épidémies d'insectes et maladies rendent l'équilibre des approvisionnements plus précaire. Il est donc impératif que les investissements en aménagement forestier soient accentués. Des résultats tangibles pourront être atteints si l'État et l'industrie acceptent qu'une partie plus considérable de leurs revenus respectifs retourne vers la forêt. Il faudra raffiner nos méthodes et concentrer nos efforts sur les sites les plus productifs. Ce faisant, je ne crois pas cependant qu'il faille remettre en cause les orientations de l'actuel régime forestier qui s'appuie, pour l'ensemble du territoire, sur une approche extensive basée sur l'utilisation prioritaire de la régénération naturelle.

\subsection{Développement de marché}

Le secteur forestier primaire, tel que nous l'avons connu jusqu'à présent, constitue l'exemple type d'un secteur à maturité.

La disponibilité de la ressource permettra encore l'ajout de quelques capacités marginales, mais rien de comparable à ce que nous avons connu par le passé. Ainsi, on pourrait assister encore à quelques transferts de la pâte meule au procédé de pâte TM, permettant un léger accroissement dans le sciage, quelques projets de OSB pourraient s'ajouter, notre capacité de MDF pourrait sans doute doubler et le secteur des pâtes et papiers pourrait prendre encore un $10 \%$ de capacité et c'est tout.

Parallèlement, nos marchés traditionnels devraient demeurer stables en terme de demande. Cependant, les prix vont continuer d'être très volatiles compte tenu de notre prédomi- nance sur le marché des commodités où nous devons subir une compétition de plus en plus vive qui nous est faite par de nouveaux arrivants ayant des échelles de coûts inférieurs aux nôtres.

Il faut, dès lors, que l'industrie forestière dans son ensemble, ainsi que chaque secteur et entreprise individuellement, se dote de stratégies commerciales qui devront s'articuler autour d'une diversification des produits et des marchés.

\section{$1^{\circ}$ La diversification des produits}

Il faut tenter de s'éloigner des commodités que d'autres arrivent à faire mieux que nous et qui commandent des échelles de prix inférieures. Pour ces produits, nous devons constater que nous sommes, à l'échelle internationale, relativement petits, et qu'il s'agit de secteurs hautement cycliques. Les produits de valeur ajoutée et ceux de seconde transformation dans des créneaux spécifiques devraient être privilégiés et ce, autant dans les produits de pâtes et papiers que les produits du bois. Le gouvernement devra, de son côté, ajuster en conséquence ses programmes de soutien.

\section{$2^{\circ}$ Diversification géographique des marchés}

L'avantage de la proximité du géant américain qui, pendant longtemps, nous a fourni l'accès facile à un marché dynamique, s'est maintenant retourné contre nous au risque même de constituer une menace pour l'assise de notre industrie forestière.

La première manifestation de cette menace a été ressentie par les producteurs de papier journal avec la venue de nouvelles capacités dans le sud des États-Unis. Par la suite, ce fut le contentieux du bois d'oeuvre avec les résultats que l'on connait maintenant. Une leçon est à retenir de ces constats: les Américains, même s'ils ont besoin de nos produits, sont aussi des producteurs et ils se sont donné des règles très efficaces pour protéger leur industrie locale de la compétition venant de l'étranger. Nous devrons donc ajuster nos comportements pour maintenir ou augmenter notre accès à ce marché dans le futur. À titre d'exemple, le dossier du bois d'oeuvre doit être considéré comme un contentieux permanent et le risque reste élevé que d'autres produits soient visés dans le futur. À la lumière de ces constats, il ne fait pas de doute que des liens stratégiques devront être entretenus avec nos voisins du Sud et ce, à tous les niveaux, incluant le consommateur.

Choisissant la voie de la facilité, nous nous sommes donc endormis au plan de la diversification de nos marchés, dépendant ainsi majoritairement du marché américain. Maintenant que ce marché semble présenter des difficultés d'accès pour le futur, nous devons constater que le train est déjà passablement en route. Malgré que nous ayons encore une bonne base de marché pour les pâtes et papiers en Europe, cette position aurait pu s'améliorer au lieu de se détériorer légèrement. Au niveau du bois d'oeuvre, nous avons mis une quinzaine d'années d'efforts pour pousser nos ventes jusqu'à un sommet historique de $400 \mathrm{Mpmp}$ pour nous situer à moins de $100 \mathrm{Mpmp}$ maintenant. De même, nous avons jusqu'à présent que timidement jeté un regard vers la force commerciale montante de l'Asie de l'Est, laissant ainsi la place à d'autres.

Pour réussir un tel virage, l'effort demandé est considérable. Il faut changer nos façons de travailler. Il faut se doter de stratégies et développer des alliances. Des efforts devront être faits pour développer une approche client et valoriser nos produits avec un souci de la qualité. Voilà un plan de travail exigeant. 


\subsection{La compétitivité}

Je l'ai mentionné précédemment, ce n'est pas tout de fabriquer le bon produit et de pouvoir le livrer au client qui en a besoin. Nous ne sommes plus les seuls à pouvoir le faire. La règle maintenant, c'est la compétitivité, c'est-à-dire l'avantage que procure la meilleure qualité au moindre coût.

\subsubsection{Action des gouvernements. Je sais que l'impact de} l'action gouvemementale sur la productivité des industries exportatrices constitue un credo cher au secteur industriel. Je puis vous affirmer qu'il s'agit là d'une préoccupation véritable du gouvemement du Québec qui a d'ailleurs été confirmée par un engagement ferme du Premier ministre lors du dernier Sommet sur l'économie et l'emploi. De façon concrète, cet engagement se traduira dans le futur par l'obligation d'évaluer l'impact, en terme de coût pour les entreprises, de toutes nouvelles règles, normes ou politiques et de faire état de l'évaluation des solutions de rechange non réglementaires.

Évidemment, on pourrait discuter longuement de tout le champ d'intervention gouvernementale, incluant la fiscalité. Je limiterai ici mon intervention au domaine plus spécifique du champ de juridiction du ministère des Ressources naturelles.

On nous a souvent rappelé l'encadrement rigide du régime forestier québécois. Comme je l'ai déjà exprimé sur d'autres tribunes, je pense que c'était le prix à payer pour une transition harmonieuse vers un nouveau régime forestier. Les résultats aujourd'hui nous permettent de faire face aux critiques les plus sévères. Autant pour la dimension «qualité» de l'aménagement que «protection» de l'environnement, aujourd'hui l'ensemble des actions de l'industrie forestière est observé par des milliers d'yeux et la moindre bavure peut se payer chèrement.

Ce constat étant fait, je pense qu'il faut reconnaître que notre industrie forestière a maintenant atteint, dans l'ensemble, un certain niveau de maturité et que les attitudes ont changé. Il y a maintenant, de la part du MRN, une volonté réelle d'assouplir l'ensemble des règles auxquelles est soumise l'industrie forestière. Nous visons dans le futur à axer notre intervention sur la mesure des résultats plutôt que sur les moyens. Il faudra à cet effet développer et utiliser de nouveaux outils. Il faudra accentuer les démarches de formation et de sensibilisation et faire une place aux interventions de vérification (audits) et de certification.

Autres aspects de l'action gouvernemenale qui peuvent affecter la compétitivité: ce sont les questions de la pertinence et du timing de ses interventions. Est-ce que l'ampleur de la mesure est conforme à l'importance du problème à résoudre? À cet égard, je pense que vous avez une responsabilité, autant sur le plan de la qualité de vos pratiques que de vos efforts à les faire connaître. Que de fois n'a-t-on vu un dossier individuel provoquant dans l'opinion publique des réactions démesurées, commandant de la part des gouvernements des mesures correctives!

Finalement, la question du timing. Il faut répondre à temps aux aspirations de la population ou aux exigences du marché. Le défi est de trouver l'équilibre entre le besoin de la mesure, l'avantage comparatif qu'il peut nous procurer ou la réduction de compétitivité qu'il peut entraîner. De même, dans la perception des redevances forestières, il faut viser à percevoir la juste valeur marchande de la ressource mais encore faut-il le faire au rythme de l'évolution des cycles du marché en réduisant le décalage entre la lecture du marché et la perception de redevances. C'est ce que nous tentons d'améliorer d'année en année.
2.3.2 La recherche et le développement. Qui dit compétitivité, dit meilleure performance, laquelle doit s'appuyer sur les meilleures techniques et technologies. Ce n'est pas l'effet du hasard si les pays présentant les meilleures performances économiques sont aussi ceux qui consacrent une part plus significative de leur PIB à la recherche et au développement. Tout le monde le sait, tout le monde le dit, mais? C'est un peu comme faire pousser des arbres, cela prend une certaine vision de l'avenir.

Je l'ai mentionné précédemment, l'effort consenti au Québec et au Canada en général nous place en position défavorable par rapport à nos principaux compétiteurs. Nous avons au Québec une des meilleures infrastructures de recherche et développement, que ce soit pour les produits du bois, des pâtes et papiers et des ressources forestières. Bien que le gouvernement du Québec ait toujours supporté de façon significative l'effort de recherche, la situation des finances publiques fragilise ses interventions pour le futur, à court terme. L'industrie se doit de prendre la relève en partenariat avec le gouvernement. Selon moi, un des meilleurs moyens d'inciter le gouvernement à maintenir ou accentuer son effort à cet effet passe par un engagement clair du secteur industriel à s'impliquer et à soutenir la recherche et le développement.

Mais il y a plus encore. Il faut inculquer dans les organisations cette culture de l'innovation technologique, cette recherche de l'avantage comparatif. Il faut mettre en contact plus étroit les gestionnaires et les chercheurs.

\subsubsection{L'investissement. Sans vouloir rebrasser de vieux dis-} cours, on a souvent reproché à votre secteur industriel d'avoir négligé le réinvestissement d'une partie des revenus pour améliorer les infrastructures afin de les maintenir à un niveau compétitif. Bien qu'un tel jugement soit injuste pour certains, il faut reconnaître que les agissements passés à cet égard font partie de la problématique avec laquelle, plusieurs d'entre vous, êtes aujourd'hui confrontés.

Sur le plan d'une certaine éthique, il apparait tout à fait inacceptable qu'une partie des revenus générés par l'utilisation des ressources naturelles appartenant à l'ensemble des citoyens ne puisse pas être réinvestie pour assurer la stabilité des emplois et des collectivités qui en dépendent. Plusieurs d'entre vous avez sans doute à l'esprit la déroute de certaines communautés ayant eu à subir la fermeture de la seule entreprise de leur milieu voyant ainsi s'évanouir l'investissement de toute une vie et la confiance mise dans cette entreprise. Certains d'entre vous avez d'ailleurs courageusement pris la relève suite à ces situations, mais dans ces cas, c'est comme partir avec deux prises.

Au-delà de ces considérations, sans des investissements judicieux et au bon moment, il faut entrevoir pour notre industrie des jours sombres. Je l'ai mentionné précédemment, on n'est plus seuls. Il s'agit d'un des plus importants paramètres où vous avez l'entière latitude et liberté d'action. Ce n'ẹt pas le cas pour la fibre, le coût de l'énergie ou de la main d'oeuvre. Couplé avec un effort approprié en R/D, l'investissement constitue votre carte maîtresse.

Encore ici, je crois que le gouvernement devra agir en partenaire avec vous. Malheureusement, le temps est révolu où on doit considérer comme une chose acquise que chaque dollar d'investissement doive être couplé par une subvention gouvernementale. Les règles du commerce international ne le permettent plus, tout comme la capacité financière de l'etat l'action 
future du governement et de ses leviers agissant, telles les sociétés d'État, devra prendre en considération la capacité financière de l'entreprise en regard du niveau de l'investissement, le degré de risque relié au caractère novateur du projet. L'intervention du gouvernement s'établira dorénavant sur un partage du risque et ultimement, un partage raisonnable du bénéfice issu de sa participation afin d'alimenter sa capacité future d'intervention. Voilà les paramètres que nous visons pour nos futures actions.

\subsubsection{Les accords internationaux qui influencent la compéti-} tivité. Vos marchés, plus que jamais, seront à l'échelle de la planète. De plus en plus, on assiste à la mise en place de règles et d'accords internationaux régissant ou influençant les marchés. Ils peuvent être à caractère purement commercial comme ceux du GATT ou à caractère environnemental comme la question des critères et indicateurs de développement durable ou une éventuelle convention internationale en matière de foresterie.

Ces règles continuent d'être en constante évolution. À titre d'exemple, l'Organisation mondiale du commerce doit aborder dans son plan de travail des questions touchant l'environnement et l'investissement. Il s'agit là d'éléments d'une importance stratégique pour votre industrie. De même, l'équilibre des règles de gestion forestière entre les différents pays aura une influence directe sur la compétitivé des pays l'un par rapport à l'autre.

Il est d'ores et déjà crucial que votre industrie s'occupe de ces questions et qu'elle puisse faire entendre sa voix. Encore ici, une action concertée des gouvernements et de l'industrie est essentielle. L'environnement commercial de demain est en train de se façonner. N'a-t-on pas laissé trop souvent au seul gouvernement le soin de débattre de ces questions?

\section{Conclusion}

Comme mentionné précédemment, les défis à relever sont nombreux et de taille. Le ministère des Ressources naturelles est prêt à partager ces défis, dans le cadre de ses mandats et dans la mesure de ses moyens d'action. Sa mission gouvernementale l'oblige à intégrer à la fois les dimensions économique, sociale et environnementale en matière de gestion forestière.

À cet effet, il doit s'assurer que son action se traduit par des conditions permettant le développement d'un secteur industriel forestier compétitif qui soit en mesure de générer le plus de retombées possibles pour le Québec. Parallèlement, il doit aussi s'assurer que sa gestion prenne en compte l'ensemble des préoccupations et des aspirations de la population en matière de gestion des ressources du milieu forestier. Finalement, il doit garantir que les interventions en milieu forestier respectent l'intégrité environnementale.

En gros, il s'agit de la base même des principes du développement durable, principes que doit aussi partager l'industrie forestière. C'est sur cette base que le gouvernement devra travailler en partenariat avec l'industrie. Cependant, la notion de «partenariat» ne signifie pas que le gouvernement est prêt à subordonner ses responsabilités de fiduciaire de la population du Québec, propriétaire de cette ressource collective, aux seuls intérêts de l'industrie ou de tout autre groupe.

Ce partenariat s'inscrit d'abord au plan de la gestion forestière, l'industrie ayant maintenant la responsabilité d'aménager la forêt, le gouvernement acceptant d'en partager le coût et la gestion. Nous sommes maintenant sur la voie de franchir un pas de plus dans cette direction puisque avec la création du fonds forestier, l'industrie partagera aussi, dans l'avenir, les responsabilités en matière de recherche et développement, production de plants pour le reboisement et inventaire forestier. Il s'agit là de responsabilités essentielles pour le maintien d'un régime forestier qui va dans le sens du développement durable.

En matière de développement économique et commercial, le ministère des Ressources naturelles entend continuer à soutenir son industrie, d'abord par la participation à la défense de ses intérêts commerciaux, que ce soit en matière de libre accès aux marchés, la promotion de l'image de notre saine gestion forestière, le développement de marchés ou la promotion de nos produits. Le financement de bureaux de promotion à l'étranger, l'engagement d'un délégué commercial à Tokyo dédié exclusivement aux produits du bois, ne sont que quelques exemples de ces actions.

De même, nous entendons continuer à soutenir, par nos programmes, l'investissement et l'innovation technologique. Comme mentionné précédemment, toute intervention gouvernementale se fera dans le futur sur une base d'affaires et de partage de risques.

Nous entendons, de plus, accompagner l'industrie dans le développement de nouveaux partenariats avec les Autochtones et les milieux locaux. L'industrie forestière étant maintenant omniprésente sur le territoire, les communautés locales veulent être associées au développement de leur territoire en vue de retirer le plus grand bénéfice possible de la gestion de l'ensemble des ressources du milieu forestier. C'est sur cette base que devrait s'appuyer d'ailleurs une éventuelle politique de forêt habitée.

Finalement, la multitude des changements dont il a été fait état dans cet exposé commande que soient revus et remis à jour plusieurs des éléments du régime forestier québécois. C'est une tâche à laquelle est aussi associée l'industrie forestière. 